


\section{Modeling Smoke Movement through Compartmented Structures}

Walter W. Jones

Glenn P. Forney

July 8, 1992

Building and Fire Research Laboratory

National Institute of Standards and Technology

Gaithersburg, MD 20899

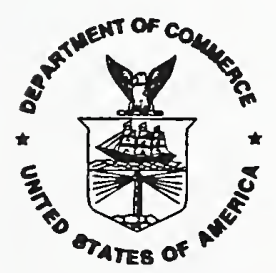

U.S. Department of Commerce

Barbara Franklin, Secretary

Technology Administration

Robert M. White, Under Secretary for Technology

National Institute of Standards and Technology

John W. Lyons, Director 


\section{Contents}

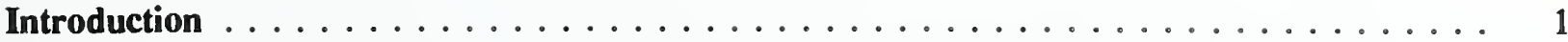

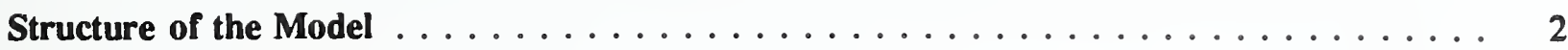

The Predictive Equations $\ldots \ldots \ldots \ldots \ldots \ldots \ldots \ldots \ldots$

Derivation of Equations for a Two-Layer Model $\ldots \ldots \ldots \ldots \ldots$

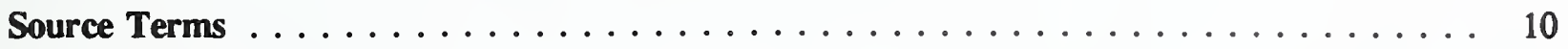

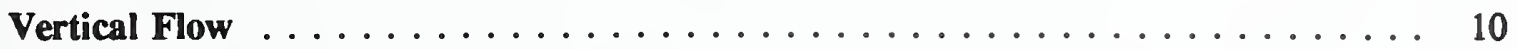

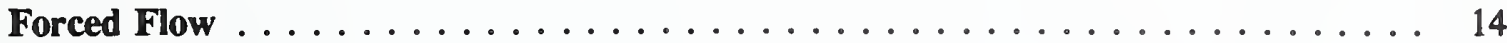

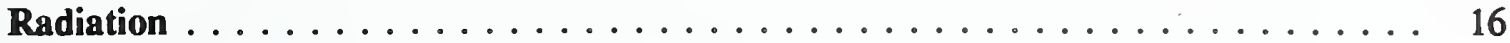

Theoretical Predictions . . . . . . . . . . . . . . . . . . . . . . . . . . 19

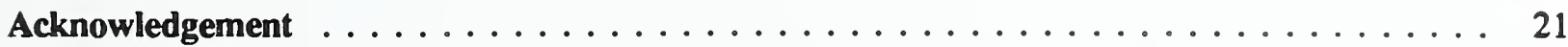

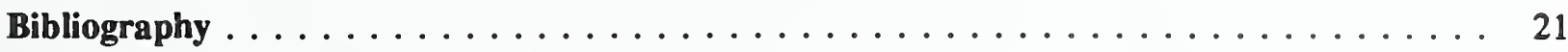


. 
Modeling Smoke Movement through Compartmented Structures

\author{
by \\ Walter W. Jones \\ and \\ Glenn P. Forney
}

This paper describes improvements which have been made in the CFAST model of fire growth and smoke transport for compartmented structures. In particular, we are interested in the ability to model the movement of toxic gases from the room of origin of a fire to a distant compartment. The newest phenomena in the model are vertical flow and mechanical ventilation. Finally, we have improved the radiation transport scheme which affects energy distribution, and therefore the buoyancy forces. These are very important in actual situations relevant to fire growth and smoke propagation, as is demonstrated.

\title{
Introduction
}

Predicting the environment in a building subject to a fire is a complex undertaking. Time scales vary from picosecond times for molecular interactions to hours for collapse of building barriers. Space scales vary from millimeters to tens of meters. To account for these broad ranges in a practical way, we use a simplification known as a zone model. A zone model is a particular implementation of the class of mathematical models known as finite element models. The concept of a zone or control volume model was pioneered by Kawagoe $^{1}$. This model embodied several approximations which reduce the computational complexity without unduly sacrificing accuracy. However, the first true multicompartment model of this type was formulated by Tanaka ${ }^{2}$. The important approximation which Tanaka stated is that flow generally occurs between like atmospheres. In other words, vent gases are assumed to flow between adjacent lower layers or upper layers. Although a drastic simplification, this rule works surprisingly well.

We have developed a deterministic model, CFAST ${ }^{3,4,5}$, which has built on this prior work, adding greater versatility while retaining the basic tenets of the zone model. For example, the lower layer is treated just like the upper layer in that it can gain and absorb energy and thus change temperature. However, the most important advance incorporated into the CFAST model is that the conservation equations are solved in their original differential form. The pressure is not assumed to be in steady state, nor the lower layer temperature to be at ambient conditions. As will be seen later, this form provides several benefits, one of which is the luxury of adding physical processes simply by adding to the 
source terms for the various predicted quantities. It also provides a model which will work over a much wider range of initial conditions.

The emphasis in this paper is on the improvements which have been made to include phenomena which have been observed experimentally ${ }^{6,7}$, but which have not been incorporated in prior models of smoke spread. Further motivation to improve the model is supplied by experience in its use in reconstructing the original path of fire growth and smoke movement in fire incidents. From these real world experiences there exists a great deal of anecdotal evidence that the model works well. Much of this latter comes from liability adjudication, fire reconstruction and product testing. As might be expected, many of these comparisons are unavailable for citation, although a recent case ${ }^{8}$ is illustrative. Nevertheless, we use these citations as confirmation of the fundamental correctness of the zone model concept, and this implementation in particular. However, these comparisons also reveal phenomena which are lacking.

We show some calculations which demonstrate these changes. One improvement which will not be discussed in this paper, but is significant in the development of such models is an improved numerical scheme ${ }^{9}$. The speed improvement is typically two to ten times faster than $\mathrm{FAST}^{3}$, its predecessor. It also solves the pressure equation completely, with no damping as was done in FAST.

We begin with a brief description of the predictive equations contained in the original model. This is done to provide a basis for discussion. In the interest of clarity and completeness, some of the earlier derivations are included. The conservation equations are turned into predictive equations for the sensible variables. The right hand side of these equations, the source terms discussed below, are the forcing functions for the ordinary differential equations. The term forcing function is used in the mathematical sense of the right hand side of an ordinary differential equation ${ }^{10}$. The refinements are discussed in terms of the original formulation of the source terms for these predictive equations. Finally we show some sample calculations to demonstrate how the refinements and improvements have affected the model from a theoretical standpoint.

\section{Structure of the Model}

The primary element of a zone model is the compartment. To form a complete model of a building or ship, many of these compartments are then strung together. The primary interest lies in the environment within each of these compartments. The basis for the model is the set of conservation equations for mass, energy and momentum for each zone. The conservation equations are recast into predictive equations for sensible variables. The set are the "natural" variables such as temperature, pressure, etc., in the compartment. Any complete set could be used, as long as we are careful not to over specify the system. The predictive equations for these variables in each compartment are then derived from the conservation equations, an equation of state and the boundary conditions to which each compartment is subject. They form a set of ordinary differential equations, whose forcing functions are the physical sources of energy and mass. 
Each compartment is subdivided into "control volumes," or zones. The premise is that the details which occur within such a volume do not concern us (at present), but the interaction between the compartments does. At present we use only two zones per compartment. There are two observations which justify this choice. Firstly, compartment fire tests usually show two distinct volumes: a hot smokey upper layer, and a relatively cool, clear lower layer. Occasionally, there are indications of a third and fourth layer where the temperature varies rapidly. Such a situation usually arises when there is strong counterflow at a vent, but normally the volume of the region, and the mass and energy contained in such a region is small. Secondly, there is reasonably good agreement between theory and experiment for the choice of two zones. Assumptions other than that of only two zones put a more severe constraint on the validity of the model. For example, the zone model concept breaks down in long corridors, where the length to width ratio is large. In order to solve this problem correctly, the horizontal momentum equation must be included in the equation set, as will be reported shortly.

Conservation of mass and energy is applied to each zone. This yields eight variables for each compartment. They are the temperature, energy, mass and pressure of each zone, with two zones per compartment. Conservation of mass and energy for each zone account for four of the required constraints. The equation of state of an ideal gas is applied to each layer for two more. Constancy of the total volume of a compartment, $\mathrm{V}_{\mathrm{u}}+\mathrm{V}_{1}=\mathrm{V}$, accounts for one more. Finally, we assume the pressure of the two zones at their interface to be the same in magnitude, $\mathrm{P}_{\mathrm{u}} \approx \mathrm{P}_{1}$. As stated previously, we assume there is no velocity within a zone or between zones within a compartment, only between compartments.

The assumption used for simplifying the pressure equation is somewhat more complex than stated above. The actual pressure consists of three parts: the base or reference (absolute) pressure, a hydrostatic term, and a fluctuation in space. Put in concrete terms, atmospheric pressure is about $10^{5} \mathrm{~Pa}$ or one bar, hydrostatic variations are 10 to $100 \mathrm{~Pa}$ $(\approx 10 \mathrm{~Pa}$ per meter), and very loud acoustic waves of about $1 \mathrm{~Pa}$. Neither the hydrostatic or fluctuations are significant in comparison with the base pressure, and fluctuations are not significant in comparison with the hydrostatic term. The base pressure is calculated at the floor of the compartment, using the conservation of energy and the equation of state. The hydrostatic term and local pressure gradients are ignored in this calculation, giving us a single pressure equation for the compartment. The momentum in the system is confined to flow through vents. It is calculated by an integral form of Euler's equation for the velocity field, namely Bernoulli's equation. Since momentum is not followed within a compartment, the implied assumption is that both horizontal and vertical velocities dissipate. The overall system, or environment, picks up the change in momentum. However, the hydrostatic term is important in calculating pressure differences across openings between compartments.

Physically a stratified medium can support both acoustic and gravity waves. Gravity waves in this context are the result of the restoring force exerted by gravity on a light fluid on top of a heavy fluid, where the depth of the heavy fluid is small. Waves in the ocean are similar. These waves do not materially influence the phenomena of interest at present (e.g. flow), but if one did not exclude them from a model, they would put a constraint on the time step allowed. Our stratagem eliminates this type of wave motion, at least for individual compartments, and thereby allows a much larger time step. Acoustic waves are eliminated 
by ignoring the momentum of the interface. Gravity waves are eliminated by the assumption of a single pressure in the compartment (no fluctuations).

The volume of a zone is calculated explicitly from the predictive equations, given the constraint of constancy of total volume of a compartment. There is no inherent three dimensional information in a finite element model. The geometric information is contained in features such as the flow of mass from compartment to compartment. The flow between compartments depends on the height of a connection, which embodies the three dimensional aspects of our problem. This requires us to delineate a relationship between the volume and the height of the zone. The model does this through the usual integral of area of cross section over height

$$
V_{u}=\int_{z_{1}}^{z_{2}} A(Z) d Z
$$

Since we have no constraints on the form this integral takes, we are free to include arbitrary area relationships. At present we make the usual assumption of rectangular parallelpipeds, but the model does the actual inversion, so changing this to suit the environment is more a matter of specification (for example, how to specify an atrium), and computation time than difficulty in the implementation.

\section{The Predictive Equations}

All current zone fire models take the mathematical form of an initial value problem for a system of differential equations. These equations are derived from the conservation of mass, energy and momentum. Subsidiary equations are the ideal gas law, and definitions of density and internal energy (for example, see ${ }^{11}$ ). These conservation laws are invoked for each zone or control volume. The implications for various choices are discussed by Forney and Moss ${ }^{12}$.

The basic element of one of these models is a zone. The basic assumption of a zone model is that properties such as temperature can be approximated throughout the zone by some uniform function. The usual approximation is that temperature, density and so on are uniform within a zone. This is not a necessary approximation. For example, a temperature which increases monotonically from the bottom of the zone to the top uniformly would, perhaps, improve the precision somewhat. However, the assumption of uniform properties is reasonable and yields good agreement with experiment. In general, these zones are grouped within compartments. The usual grouping is two gas layers per compartment. Once again, more could be utilized with a concomitant increase in computing time, but little improvement in accuracy. There are two conjectures which are made which are reasonable and dramatically improve the ease of solving these equations. Momentum is ignored within a compartment. The momentum of the interface has no significance in the present context. However, at boundaries such as windows, doors and so on, the Euler equation is integrated explicitly to yield the Bernoulli equation. This is solved implicitly in the equations which are discussed below. This stratagem avoids the short time step imposed by acoustic waves 
(Courant condition), which couple the pressure equation and the momentum equation.

The other approximation is that the pressure is approximately uniform within a compartment. The argument is that a change in pressure of a few tens of Pascals over the height of the compartment is negligible in comparison with atmospheric pressure. Once again, this is applied to the basic conservation equations. This is consistent with the point source view of finite element models. Volume is merely one of the dependent variables. However, the hydrostatic variation in pressure is taken into account in calculating pressure differences between compartments, and for variations in height across vents.

Many formulations based upon these assumptions can be derived. Several of these are discussed later. One formulation can be converted into another using definitions of density, internal energy and the ideal gas law. Though equivalent analytically, these formulations differ in their numerical properties. Also, until the development of FAST [3], all models of this type assumed that the pressure equilibrated instantaneously, and thus the $\mathrm{dP} / \mathrm{dt}$ term could be set to zero. This was an attempt to solve the numerical problem known as stiffness. The time for significant change in each of the variables is significantly different for each equation. This is particularly acute for the pressure equation. It is not a matter of equilibration of the density or pressure within the compartment. Rather it is how strong the coupling is between the time rate of change of the variable ( $\mathrm{dP} / \mathrm{dt}$ for example), and the forcing function, or right hand side of the predictive equation. Writing each of the predictive equations in the form

$$
\frac{d x}{x}=A d \tau,
$$

the coefficient A varies by orders of magnitude amongst the equations. Typically, the ratio of these coefficients for the pressure to any other variable is $\approx c_{p}$, or about 1000 . By setting the $\mathrm{dP} / \mathrm{dt}$ term to zero, this difference vanishes. However, as has been shown ${ }^{13}$, it is much easier to solve these equations in the differential than the algebraic form if the proper solver is used.

Each formulation can be expressed in terms of mass and enthalpy flow. These rates represent the exchange of mass and energy between zones due to physical phenomena such as plumes, natural and forced ventilation, convective and radiative heat transfer, and so on. For example, a vent exchanges mass and energy between zones in connected rooms, a fire plume typically adds heat to the upper layer and transfers entrained mass and energy from the lower to the upper layer, and convection transfers energy from the gas layers to the surrounding walls.

We use the formalism that the mass flow to the upper and lower layers is denoted $\dot{m}_{U}$ and $\dot{m}_{\mathrm{L}}$ and the enthalpy flow to the upper and lower layers is denoted $\dot{s}_{\mathrm{U}}$ and $\dot{\mathrm{s}}_{\mathrm{L}}$. It is tacitly assumed that these rates may be computed in terms of zone properties such as temperatures and densities. These rates represent the net sum of all possible sources of mass and energy due to phenomena such as those listed above. The numerical characteristics of the various formulations are easier to identify if the underlying physical phenomena are 
decoupled in this way.

Many approximations are necessary when developing physical sub-models for the mass and enthalpy terms. For example, most fire models assume that 1) the specific heat terms $c_{p}$ and $c_{v}$ are constant even though they depend upon temperature, 2) hydrostatic terms can be ignored in the equation of state (the ideal gas law) relating density of a layer with its temperature. However, the derivations which follow are all based on the basic conservation laws.

\section{Derivation of Equations for a Two-Layer Model}

We divide a compartment into two control volumes, a relatively hot upper layer and a relatively cooler lower layer. The gas in each layer has attributes of mass, internal energy, density, temperature, and volume denoted respectively by $m_{i}, E_{i}, \rho_{i}, T_{i}$, and $V_{i}$ where $i=L$ for the lower layer and $i=U$ for the upper layer. The compartment as a whole has the attribute of pressure $P$. These eleven variables are related by means of the following seven constraints

$$
\begin{aligned}
& \rho_{i}=\frac{m_{i}}{V_{i}} \quad \text { (density) } \\
& E_{i}=c_{v} m_{i} T_{i} \quad \text { (internal energy) } \\
& P=R_{\rho_{i}} T_{i} \quad \text { (ideal gas law) } \\
& V=V_{L}+V_{U} \quad \text { (total volume) }
\end{aligned}
$$

The specific heat at constant volume and at constant pressure $c_{v}$ and $c_{p}$, the universal gas constant, $R$, and the ratio of specific heats, $\gamma$, are related by $\gamma=c_{p} / c_{v}$ and $R=c_{p}-c_{v}$. For air, $c_{p} \approx 1000 \mathrm{~kJ} / \mathrm{kg} \mathrm{K}$ and $\gamma=1.4$. This leaves four unconstrained, or independent, variables. So we require four equations for a unique solution. The four are the conservation of mass and energy for each layer.

The differential equations for the mass in each layer are

$$
\begin{aligned}
& \frac{d m_{L}}{d t}=\dot{m}_{L} \\
& \frac{d m_{U}}{d t}=\dot{m}_{U}
\end{aligned}
$$

The first law of thermodynamics states that the rate of increase of internal energy plus the 
rate at which the layer does work by expansion is equal to the rate at which enthalpy is added to the gas. In differential form this is

$$
\overbrace{\frac{d E_{i}}{d t}}^{\text {internal energy }}+\overbrace{P \frac{d V_{i}}{d t}}^{\text {work }}=\underbrace{\text { enthalpy }}_{\dot{s}_{i}}
$$

A differential equation for pressure can be derived by adding the upper and lower layer versions of equation (8), noting that $d V_{U} / d t=-d V_{L} / d t$, and substituting the differential form of equation (4) to yield

$$
\frac{d P}{d r}=\frac{\gamma-1}{V}\left(\dot{s}_{L}+\dot{s}_{U}\right)
$$

Differential equations for the layer volumes can be obtained by substituting equation (4) into equation (8) to obtain

$$
\frac{d V_{i}}{d t}=\frac{1}{P \gamma}\left((\gamma-1) \dot{s}_{i}-V_{i} \frac{d P}{d t}\right)
$$

By substituting equation (10) into the differential form of equation (7), we obtain

$$
\frac{d E_{i}}{d t}=\frac{1}{\gamma}\left(\dot{s}_{i}+V_{i} \frac{d P}{d t}\right)
$$

A equation for density can be derived by applying the chain rule to $\frac{d \rho_{t}}{d t}=\frac{d}{d t}\left(\frac{m_{t}}{V_{t}}\right)$ and using equation (10) to eliminate $d V_{i} / d t$ to obtain

$$
\frac{d \rho_{i}}{d t}=-\frac{1}{c_{p} T_{i} V_{i}}\left(\left(\dot{s}_{i}-c_{p} \dot{m}_{i} T_{i}\right)-\frac{V_{i}}{\gamma-1} \frac{d P}{d t}\right) .
$$

Temperatures can be obtained from the equation of state by applying the chain rule to $\frac{d T_{t}}{d t}=\frac{d}{d t}\left(\frac{P}{R p_{1}}\right)$ and using equation (12) to eliminate $d \rho / d t$ to obtain

These equations for each of the eleven variables are summarized in Table 1. The time 


$$
\frac{d T_{i}}{d t}=\frac{1}{c_{p} p_{i} V_{i}}\left(\left(\dot{s}_{i}-c_{p} \dot{m}_{i} T_{i}\right)+V_{i} \frac{d P}{d t}\right) .
$$

evolution of these solution variables can be computed by solving the corresponding differential equations together with appropriate initial conditions. The remaining seven variables can be determined from the four independent solution variables.

There are, however, many possible differential equation formulations. Indeed, there are 330 different ways to select four variables from eleven. Many of these systems are incomplete due to the relationships that exist between the variables given in equations (3) to (6). For example the variables, $\rho_{U}, V_{U}, m_{U}$ and $P$ form a dependent set since $\rho_{U}=m_{U} /$ $V_{U}$.

The number of differential equation formulations can be considerably reduced by not mixing variable types between layers; that is, if upper iayer mass is chosen as a solution variable, then lower layer mass must also be chosen. For example, for two of the solution variables choose $m_{L}$ and $m_{U}$, or $\rho_{L}$ and $\rho_{U}$, or $T_{L}$ and $T_{U}$. For the other two solution variables pick $E_{L}$ and $E_{U}$ or $P$ and $V_{L}$ or $P$ and $V_{U}$. This reduces the number of distinct formulations to nine. Since the numerical properties of the upper layer volume equation are the same as a lower layer one, the number of distinct formulations can be reduced to six.

Table I. Conservative Zone Modeling Differential Equations

\begin{tabular}{|c|c|}
\hline Equation Type & Differential Equation \\
\hline i'th layer mass & $\frac{d m_{i}}{d t}=\dot{m}_{i}$ \\
\hline pressure & $\frac{d P}{d t}=\frac{\gamma-1}{V}\left(\dot{s}_{L}+\dot{s}_{U}\right)$ \\
\hline i'th layer energy & $\frac{d E_{i}}{d t}=\frac{1}{\gamma}\left(\dot{s}_{i}+V_{i} \frac{d P}{d t}\right)$ \\
\hline i'th layer volume & $\frac{d V_{i}}{d t}=\frac{1}{\gamma P}\left((\gamma-1) \dot{s}_{i}-V_{i} \frac{d P}{d t}\right)$ \\
\hline i'th layer density & $\frac{d \rho_{i}}{d t}=-\frac{1}{c_{p} T_{i} V_{i}}\left(\left(\dot{s}_{i}-c_{p} \dot{m}_{i} T_{i}\right)-\frac{V_{i}}{\gamma-1} \frac{d P}{d t}\right)$ \\
\hline i'th layer temperature & $\frac{d T_{i}}{d t}=\frac{1}{C_{p} p_{i} V_{i}}\left(\left(\dot{s}_{i}-c_{P} \dot{m}_{i} T_{i}\right)+V_{i} \frac{d P}{d t}\right)$ \\
\hline
\end{tabular}


The current version of CFAST is set up to use the equation set for layer temperature, layer volume, and pressure as shown in eq (14), (15), (16) and (17). However, the internal structure of the model is such that it will allow any of the formulations above to be substituted with minimal effort.

$$
\begin{aligned}
& \frac{d \Delta P}{d t}=\frac{\gamma-1}{V}\left(\dot{s}_{L}+\dot{s}_{U}\right) \\
& \frac{d V_{U}}{d t}=\frac{1}{\gamma P}\left((\gamma-1) \dot{s}_{U}-V_{U} \frac{d \Delta P}{d t}\right) \\
& \frac{d T_{U}}{d t}=\frac{1}{C_{p} \rho_{U} V_{U}}\left(\left(\dot{s}_{U}-c_{P} \dot{m}_{U} T_{U}\right)+V_{U} \frac{d \Delta P}{d t}\right) \\
& \frac{d T_{L}}{d t}=\frac{1}{C_{p} \rho_{L} V_{L}}\left(\left(\dot{s}_{L}-C_{P} \dot{m}_{L} T_{L}\right)+V_{L} \frac{d \Delta P}{d t}\right)
\end{aligned}
$$

\section{Source Terms}

The sensible variables in each compartment are described by the set of predictive equations. The form of the equations is that the physical phenomena are source terms on the right-hand-side of these equations ${ }^{3}$. Such a formulation makes the addition (and deletion) of physical phenomena and changing the form of algorithms a relatively simple matter.

The source terms important to smoke transport in buildings are radiation transfer between the zones and walls, and burning object(s), convective heating by boundaries, plume flow and vent flow, species generation and loss, and finally the fire or fires. There are subsidiary equations which must be solved also, but will not be discussed here. An example of the latter is heat conduction through partitions such as ceilings and walls. Most of the phenomena have been discussed adequately in the papers by Jones ${ }^{3,14}$ and Jones and Peacock ${ }^{4,15}$. The following three sections describe the 3 major additions to CFAST which enhance its ability to calculate the environment resulting from a fire.

\section{Vertical Flow:}

Flow through vents comes in two varieties. The first we refer to as horizontal flow. It is the flow which is normally thought of in discussing fires. It encompasses flow through doors, windows and so on. The other is vertical flow and can occur if there is a hole in the ceiling or floor of a compartment. This latter phenomena is particularly important in two disparate situations: a ship, and the role of fire fighters doing roof venting. 
Flow through normal vents is governed by the pressure difference across the vent. It is the dominant transfer mechanism in solving the conservation equations because it fluctuates the most rapidly of all the source terms and is most sensitive to changes in the environment. A momentum equation for the zone boundaries is not solved directly. Instead momentum transfer at the zone boundaries is included by using an integrated form of Euler's equation, namely Bernoulli's solution for the velocity equation. This solution is augmented for restricted openings by using flow coefficients ${ }^{16}$ to allow for constriction from finite size doors. The flow (or orifice) coefficient is an empirical term which addresses the problem of constriction of velocity streamlines at an orifice.

There are two situations which give rise to flow through vents. The first, and usually thought of in fire problems, is that of air or smoke which is driven from a compartment by buoyancy. The second type of flow is due to a piston effect which is particularly important when conditions in the fire environment are changing rapidly. Rather than depending on density differences between the two gases, the flow is forced by volumetric expansion. The earlier version of this model did not solve this part of the problem entirely correctly. In most cases the differences are small except for rapidly changing situations. However, these small differences become very important if we wish to follow flows due to small pressure differences, such as occurs in a mechanical ventilation system. Atmospheric pressure is about $100000 \mathrm{~Pa}$, fires produce pressure from 1 to $1000 \mathrm{~Pa}$ and mechanical ventilation systems typically involve pressures about 1 to $100 \mathrm{~Pa}$. In order to solve these interactions correctly, we must be able to follow pressure differences of $\approx 0.1 \mathrm{~Pa}$ out of $10^{5}$.

When dealing with flow between a compartment containing a fire and an ambient environment, there will be only a single neutral plane. A neutral plane is a point at which the flow into or out of a vent is reversed. This is the situation observed when looking at a building from the outside, for example. For flow between two compartments which contain strongly stratified atmospheres, the flow field is more complicated. It is possible to have up to three neutral planes ${ }^{4,13}$ in this situation. The model does this calculation correctly. It is done explicitly in the integral over the height of the vents, and is discussed later.

Bernoulli's equation is the integral of the Euler equation and applies to general initial and final velocities and pressures. The implication of using this equation for a zone model is that the initial velocity in the doorway is the quantity sought, and the final velocity in the target compartment vanishes. That is, the flow velocity vanishes where the final pressure is measured. Thus, the pressure at a stagnation point is used. This is consonant with the concept of uniform zones which are completely mixed and have no internal flow. The general form for the velocity of the mass flow is given by

$v=C\left(\frac{2 \delta P}{\rho}\right)^{1 / 2}$

where $\mathrm{C}$ is the constriction (or flow) coefficient $(\approx 0.7), \rho$ is the gas density on the source side, and $\delta \mathrm{P}$ is the pressure across the interface. (Note: at present we use a constant $\mathrm{C}$ for all gas temperatures) We apply the above equation to rectangular openings which allows us to remove the width from the mass flux integral. That is 
mass flux $=\int_{\text {widhh }} \int_{\text {height }} \rho v d z d w \rightarrow$ width $\int_{z_{1}}^{z_{2}} \rho v d z$

The simplest means to define the limits of integration is with neutral planes, that is the height at which flow reversal occurs, and physical boundaries such as sills and soffits. By breaking the integral into intervals defined by flow reversal, a soffit, a sill, or a zone interface, the flow equation can be integrated piecewise analytically and then summed.

The approach to calculating the flow field is of some interest. When one of the limits of integration is at a height where $\delta \mathrm{P}$ is zero (a neutral plane), the mass flow over the interval $\left(z_{2}-z_{1}\right)$ is given by

$$
\frac{2}{3} \operatorname{CS}\left(z_{2}-z_{1}\right)(2 p \delta P)^{1 / 2}
$$

where $\delta \mathrm{P}$ is the pressure difference at the other end, and for the case of no neutral plane, we obtain

$$
\dot{m}_{i-0}=\frac{2}{3} \operatorname{CS}(2 \rho)^{1 / 2}\left(z_{2}-z_{1}\right) \frac{\left(P_{2}^{3 / 2}-P_{1}^{3 / 2}\right)}{\left(P_{2}-P_{1}\right)}
$$

where $\rho$ is the average mass density within the area of flow from the source compartment. The flow will be in the opposite direction if $P_{o}>P_{i}$. The pressure at $z_{1}$ is $P_{1}$ and at $z_{2}$ is $P_{2}$. The integration is started at the lowest point at which flow can occur, the sill or floor. Then the next change point is calculated. It is either a soffit or a change in the relative gas density (the interface). Within this interval there is either a neutral plane or not. In either case, the flow equation can be integrated analytically. In the former case, the bi-directional flow is calculated from the neutral plane to the two end points. The evaluation of this function is quite fast since one of the endpoints in the integral is zero. In the latter case the solution can better be expressed as

$$
\dot{m}_{i \rightarrow 0}=\frac{2}{3} \operatorname{cs}(2 p)^{1 / 2}\left(z_{2}-z_{1}\right)\left[y+\frac{x^{2}}{x+y}\right]
$$

where

$$
\begin{aligned}
& x=P_{1}^{1 / 2} \\
& y=P_{2}^{1 / 2}
\end{aligned}
$$


The numerical evaluation of eq (22) is considerably faster than using eq (21) and does not suffer the numerical difficulty of dividing by zero as $P_{1}$ and $P_{2}$ approach each other.

A check is then made to see if there is additional space (opening above the present position) through which flow can occur. If so, then the integration process starts from the last endpoint $\left(z_{2}\right)$ and continues until the soffit is reached.

Flow through a ceiling or floor vent is somewhat more complicated than through door or window vents. The simplest form is of flow in uni-directional, driven solely by a pressure difference. This is analogous to flow in the horizontal direction driven by a piston effect of expanding gases. Once again, it can be calculated based on the Bernoulli equation, and presents little difficulty. However, in general we must deal with a much more complex situation. There are two situations that must be modeled in order to have a proper understanding of smoke movement. The first is an occurrence of puffing. When a fire exists in a compartment in which there is only a hole in the ceiling, the fire will burn until the oxygen has been depleted, pushing gas out the hole. Eventually the fire will die down. At this point ambient air will rush back in and the process will be repeated. Combustion is thus tightly coupled to the flow. The other case is exchange flow which occurs when the fluid configuration across the vent is unstable. Both of these pressure regimes require a calculation of the onset of the flow reversal mechanism.

Normally a non-zero cross vent pressure difference tends to drive unidirectional flow from the higher to the lower pressure side. An unstable fluid density configuration occurs when the pressure alone would dictate stable stratification, but the fluid densities are reversed. That is, the hotter gas is underneath the cooler gas. Flow induced by an unstable fluid density configuration tends to lead to bi-directional flow, with the fluid in the lower compartment rising into the upper compartment. This situation might arise in a real fire if the room of origin suddenly had a hole punched in the ceiling. We make no pretense of being able to do this instability calculation analytically. We use Coopers's algorithm ${ }^{17}$ for computing mass flow through ceiling and floor vents. It is based on correlations to model the unsteady component of the flow. What is surprising is that we can find a correlation at all for such a complex phenomenon. There are two components to the flow. The first is a net flow dictated by a pressure difference. The second is an exchange flow based on the relative densities of the gases. The overall flow is given by ${ }^{17}$

$\dot{m}=C f(\gamma, \varepsilon)\left(\frac{\delta P}{\bar{\rho}}\right)^{1 / 2} A_{v}$

where

$C=0.68+0.17 \varepsilon$, 
$\varepsilon=\frac{\delta P}{P}$

and $\mathrm{f}$ is a weak function of both $\gamma$ and $\varepsilon$. In the situation where we have an instability, there can be bi-directional flow. This is called the Rayleigh-Taylor instability, and is quite difficult to model. We make no attempt to do this from first principles, but rather rely on the correlations. The algorithm for this exchange flow is given by

$\dot{m}_{e x}=0.1\left(\frac{g \delta \rho A_{v}^{5 / 2}}{\rho_{a v}}\right)\left(1.0-\frac{2 A_{v}^{2} \delta \rho}{S^{2} g \delta \rho D^{5}}\right)$

where

$D=2 \sqrt{\frac{A_{v}}{\pi}}, S=\{0.754$ or 0.942$\}$

for round or square openings, respectively.

A simple example of the effect of this exchange flow can be shown with the following example. Consider two closed compartments, each $10 \mathrm{~m}$ in height, one on top of the other, connected by a one meter diameter round hole. Given hydrostatic equilibrium, there will be no flow between the compartments. By varying the pressure and density of the gas in the lower compartment very slightly, we calculate the flow between the compartments, as shown in Figure 1.

\section{Forced Flow:}

The final type of flow which is important in this type of simulation is forced flow through a duct system. The model for mechanical ventilation is based on the theory of networks. This is a simplified form of Kirchoff's law which says that flow into a node must be balanced by flow out of the node. There is a close analog to electrical networks for which the flow consists of electrons. In the case of ventilation, the flow is formed by molecules of air. The conservation equation differs slightly from that of an electrical system, but the basic ideas carry over. In the former case, we have

$$
\text { voltage }=\text { current } \times \text { resistance. }
$$

In the present case we have

pressure change $=$ mass flow $\times$ mass flow $\times$ resistance.

So the application of network theory is used, although the circuit laws are slightly different. In practice, as with the electrical analog, one solves the problem by summing all of the equations for the nodes, and require that the mass be conserved at each node. Thus we turn 
the equation around and put it into the form

mass flow $=$ conductance $\times$ (pressure drop across a resistance $)^{1 / 2}$.

For each node, this flow must sum to zero. There are several assumptions which are made in computing this flow in ducts, fans, elbow, etc. First, we assume unidirectional flow. Given the usual size of ducts, and the nominal presence of fans, this is quite reasonable. Also, the particular implementation used here ${ }^{18}$ does not allow for reverse flow in the duct system. The difficulty lies in describing how a fan behaves in such a case.

Given that we can describe mass flow in terms of pressure differences and conductance, the conservation equation for each node is

$$
\sum_{j} \dot{m}_{i, j}=0
$$

The index " $\mathrm{j}$ " is a summation over connections to a node, and there is an equation " $\mathrm{i}$ " for each node. The remaining problem is to specify the boundary conditions. At each connection to a compartment, the pressure is specified. Then, given that flow is unidirectional, the mass and enthalpy flow into or out of a room can be calculated explicitly. Thus we end up with a set of equations of the form

$$
\begin{gathered}
\mathrm{f}_{1}\left(\mathbb{P}_{1}, \mathbb{P}_{2}, \ldots\right)=0 \\
\cdot \\
f_{i}\left(\mathbb{P}_{1}, \mathbb{P}_{2}, \ldots\right)=0 \\
\cdot \\
f_{n}\left(\mathbb{P}_{1}, \mathbb{P}_{2}, \ldots\right)=0 .
\end{gathered}
$$

This is an algebraic set of equations that is solved simultaneously with the equations for flow in the compartments.

The equations describe the relationship between the pressure drop across a duct, the resistance of a duct, and the mass flow. The pressure can be changed by conditions in a compartment, or a fan in line in the duct system. Resistance arises from the finite size of ducts, roughness on surfaces, bends and joints. To carry the electrical analog a little further, fans act like constant voltage sources. The analogy breaks down, however, in that our analogous voltage and resistance are related by the square of the current, rather than being linearly proportional. Since we are using the current form of the conservation equation to balance the system, recast the flow in terms of a conductance

$$
\dot{\mathbf{m}}=\mathbf{G} \times \sqrt{\Delta \mathbf{P}} \text {. }
$$


The conductance can be expressed generally as

$G=\left(\frac{2 \rho}{C_{0}}\right)^{1 / 2} A_{0}$

where $C_{0}$ is the flow coefficient (usually a loss term), and $A_{0}$ is the area of the inlet, outlet, duct, contraction or expansion joint, coil, damper, bend, filter, and so on. Values for the most common of these items are tabulated in the ASHRAE Handbook?

Ducts are long pipes through which gases can flow. They have been studied much more extensively than other types of connections. For this reason, eq (30) can be put into a form which allows one to characterize the conductance in more detail, depending on the type of duct, such as oval, round, square, and so on. The form derives from the Darcy equation and is

$$
G=\left(\frac{P L}{2 \rho D_{e} A_{0}^{2}}\right)^{1 / 2},
$$

where $\mathrm{F}$ is the friction factor and can be calculated from

$$
\frac{1}{\sqrt{\mathrm{F}}}=-2 \log \left(\frac{\epsilon}{3.7 \mathrm{D}_{e}}+\frac{2.51}{\mathrm{R}_{e} \sqrt{\mathrm{F}}}\right)
$$

For each node in the system, one has an entry of the form of eq (35). This set of equations is then solved at each time step. In the present form, the solution to the duct system is split from that of the buoyancy driven flow. This is justified based on the long time constant for change of the flow pattern in such a system. Implicit in this assumption is that there is only a very weak interaction between the systems of equations. When we begin to deal with the problem of flow reversal then the fan characteristics will be coupled much more closely with the buoyancy driven flow and we will have to reformulate the solution.

\section{Radiation:}

The purpose of the new radiation algorithm is to enhance the radiative module to allow the ceiling, the upper wall segments, the lower wall segments and the floor to transfer radiant heat independently and consistently. The original radiation algorithm used the extended floor and ceiling concept for computing radiative heat exchange. The room was assumed to consist of two wall segments: an extended ceiling and an extended floor. The extended ceiling consisted of the ceiling plus the upper wall segments. Similarly, the extended floor consisted of the floor plus the lower wall segments. The upper layer was modeled as a sphere equal in volume to the volume of the upper layer. Radiative heat 
transfer to and from the lower layer was ignored. This concept is inconsistent with the way heat conduction is handled, since we solve up to four heat conduction problems for each room: the ceiling, the upper wall, the lower wall and the floor.

To calculate the radiation absorbed in a zone, a heat balance must be done which includes all surfaces which radiate to and absorb radiation from a zone. The form of the terms which contribute heat to an absorbing layer are the same for all layers. Essentially we assume that all zones in these models are similar so we can discuss them in terms of a general layer contribution. For this calculation to be done in a time commensurate with the other sources, some approximations are necessary.

Radiation can leave a layer by going to another layer, by going to the walls, by exiting through a vent, by heating an object, or by changing the pyrolysis rate of the fuel source. Similarly, a layer can be heated by absorption of radiation from these surfaces and objects as well as from the fire itself. The formalism which we employ for the geometry and view factor calculation is that of Siegel and Howell ${ }^{20}$. Although the radiation could be done with a great deal of generality, we have assumed that the zones and surfaces radiate and absorb like a grey body.

Radiation is an important mechanism for heat exchange in compartments subject to fires. It is important in the present application because it can affect the temperature distribution within a compartment, and thus the buoyancy forces. In the present implementation the fire is assumed to be a point source. It is also assumed that plumes do not radiate. We use a simplified geometrical equivalent of the compartment in order to calculate the radiative transfer between the ceiling, floor and layer(s). The original paper which described FAST pointed out that there was an inconsistency in the interaction between the walls and the radiation from and to the gas layers. This modification fixes that problem. A radiative heat transfer calculation could easily dominate the computation in any fire model. This is because radiation exchange is a global phenomena. Each portion of an enclosure interacts radiatively with every other portion that it "sees." Therefor it is important to construct algorithms for radiative heat transfer that are both accurate and efficient ${ }^{19}$.

This is a "next step" algorithm for computing radiative heat transfer between the bounding surfaces of a compartment containing upper and lower layer gasses and point source fires. The two wall radiation model used has been enhanced to treat lower layer heating and to treat radiative heat exchange with the upper and lower walls independently of the floor and ceiling. We refer to this as the four wall model.

The four wall algorithm for computing radiative heat exchange is based upon the equations developed in Siegel and Howell ${ }^{20}$ which in turn is based on the work of Hottel $^{21}$. Siegel and Howell model an enclosure with $\mathrm{N}$ wall segments and an interior gas. A radiation algorithm for a two layer zone fire model requires treatment of an enclosure with two uniform gases. Hottel and Cohen ${ }^{22}$ developed a method where the enclosure is divided into a number of wall and gas volume elements. An energy balance is written for each element. Each balance includes interactions with all other elements. Treatment of the fire and the interaction of the fire and gas layers with the walls is based upon the work of Yamada and Cooper ${ }^{23}$. They model fires as point heat sources radiating uniformly in all 
directions and use the Lambert-Beer law to model the interaction between heat emitting elements (fires, walls, gas layers) and the gas layers. The original formulation is for an $\mathbf{N}$ wall configuration. Even the more modest approach of a four wall configuration for computing radiative heat transfer is more sophisticated than was used previously. By implementing a four-wall rather than an $\mathrm{N}$-wall model, significant algorithmic speed increases were achieved. This was done by exploiting the simpler structure of the four wall problem.

The radiation exchange at the k'th surface is shown schematically in Figure 2. For each wall segment $\mathrm{k}$ from 1 to $\mathrm{N}$ we must find a net heat flux, $\Delta \mathrm{q}_{\mathrm{k}}{ }^{\prime}$, such that

$$
A_{k} \sigma_{k} T_{k}^{4} q_{k}^{i n}+\left(1-\epsilon_{k}\right) q_{k}^{i n}=q_{k}^{i n}+A_{k} \Delta q_{k}^{\prime \prime}(k=1, . . N)
$$

Radiation exchange at each wall segment has emitted, reflected, incoming and net radiation terms. Equation (36) then represents a system of linear equations that must be solved for $\Delta q^{\prime \prime}$ to determine the net fluxes given off by each surface. Finding a solution of this linear system is the bulk of the work required to implement the net radiation method of Siegel and Howell. Equation (37) derived by Siegel and Howell ${ }^{20}$ and listed there as equations (17-20), is called the net radiation equation,

$$
\frac{\Delta q_{k}^{\prime \prime}}{\epsilon_{k}}-\sum_{j=1}^{N} \frac{1-\epsilon}{\epsilon_{j}} \Delta q_{j}^{\prime \prime} F_{k-j} \tau_{j-k}=\sigma T_{k}^{4}-\sum_{j=1}^{N} \sigma T_{j}^{4} F_{k-j} \tau_{j-k}-\frac{c_{k}}{A_{k}} .
$$

where $\sigma$ is the Stefan-Boltzman constant, $\varepsilon_{k}$ is the emissivity of the k'th wall segment, $T_{k}$ is the temperature of the $\mathrm{k}^{\prime}$ th wall segment, $\mathrm{F}_{\mathrm{k}-\mathrm{j}}$ is a configuration factor, and $\tau$ is a transmissivity factor. This latter is the fraction of energy passing unimpeded through a gas along a path from surface $j$ to $k$. The parameters $c_{k}$ represent the various sources of heat, namely the fire itself and the gas layers. In the form shown, the view factor of the $k$ 'th element is included in the parameter $\mathrm{c}$.

The actual implementation uses a slightly modified form of equation (37), namely

$$
\begin{aligned}
& \Delta \hat{q}_{k}^{\prime \prime}-\sum_{j=1}^{N}\left(1-\epsilon_{j}\right) \Delta \hat{q}_{j}^{\prime \prime} F_{k-j} \tau_{j-k}=\sigma T_{j}^{4}-\sum_{j=1}^{N} \sigma T_{j}^{4} F_{k-j} \tau_{j-k}-\frac{c_{k}}{A_{k}}, \text { where } \\
& \Delta q_{k}^{\prime \prime}=\epsilon_{k} \Delta \hat{q}_{k}^{\prime \prime} .
\end{aligned}
$$

There are two reasons for solving equation (38) rather than equation (37). First, since $\varepsilon_{\mathrm{k}}$ does not occur in the denominator, radiation exchange can be calculated when some of the wall segments have zero emissivity. Second and more importantly, the matrix corresponding to the linear system of equation (39) is diagonally dominant ${ }^{19}$. Iterative algorithms can be used to solve such systems more efficiently than direct methods such as Gaussian elimination. Diagonal dominance will occur as the emissivity approaches unity. Typical values of the 
emissivity for walls subject to a fire environment are in the range of $0.85<\varepsilon<0.95$, so this is a reasonable approximation. For those cases where diagonal dominance does not hold, the calculation takes longer. The computation of, $F_{k-j}, \tau_{j-k}$ and $c_{k}$ is discussed by Forney ${ }^{19}$. It is shown how it is possible to use the symmetries present in the four wall segment problem to minimize the number of direct configuration factor calculations required.

CFAST models the temperature of the four wall segments independently. A two wall model for radiation exchange can break down when the temperatures of the ceiling and upper walls differ significantly. This could happen in the mode when different wall materials are used as boundaries for the ceiling, walls and floor. To demonstrate this, consider the following example.

To simplify the comparison between the two and four wall segment models, assume that the wall segments are black bodies (the emissivities of all wall segments are one) and the gas layers are transparent (the gas absorptivities are zero). This is legitimate since for this example we are only interested in comparing how a two wall and a four wall radiation algorithm transfer heat to wall segments. Let the room dimensions be $4 \times 4 \times 4$ [m], the temperature of the floor and the lower and upper walls be $300 \mathrm{~K}$. Let the ceiling temperature vary from $300 \mathrm{~K}$ to $600 \mathrm{~K}$.

Figure 3 shows a plot of the heat flux striking the ceiling and upper wall as a function of the ceiling temperature. The two wall model predicts that the extended ceiling (a surface formed by combining the ceiling and upper wall into one wall segment) cools, while the four wall model predicts that the ceiling cools and the upper wall warms. The four-wall model moderates temperature differences that may exist between the ceiling and upper wall (or floor and lower wall) by allowing heat transfer to occur between the ceiling and upper wall. The two wall model is unable to predict heat transfer between the ceiling and the upper wall since it models them both as one wall segment.

\section{Theoretical Predictions}

An appreciation of the relative effect of each of these various mechanisms to influence the environment is important to simulating fires in actual circumstances. The comparison is of the various types of flow which can occur in a building. The starting point is the relative size of each of these types of flow. The physical situation is chosen to demonstrate the importance of each phenomenon, and is based on physical situations that actually arise in the environments in which we are interested.

The calculations which follow are based on the two compartments shown in Figure 4. The comparison is of the vent flow, so the physical parameters were chosen to yield flows of approximately equal magnitude. The fire used was a constant $25 \mathrm{~kW}$. The absolute height of the floor of the second compartment is 2.3 meters, so it coincides with the ceiling of the first compartment. There is a door from the first compartment $\left(1.07 \times 1 \mathrm{~m}^{2}\right)$ to the outside, and a window $\left(1.07 \times 1 \mathrm{~m}^{2}\right)$ from the second compartment to the outside. The comparison is for flow through normal vents, through a vertical vent $(0.34 \mathrm{~m}$ diameter $)$, a duct $(0.1 \mathrm{~m}$ diameter) with no fan and finally a fan system (fan flow is $0.143 \mathrm{~m}^{3} / \mathrm{s}$ ). The cases are 
1) door only from compartment 1 to the outside

2) no door, a hole in the ceiling/floor between 1 and 2, window from 2 to the outside

3 ) door from 1 to the outside, duct work from 1 to 2, window from 2 to the outside

4) door from 1 to the outside, duct system with a fan from 1 to 2 , window from 2 to the outside.

The comparison is of the vent flow, so the physical parameters were chosen to yield flows of approximately equal magnitude. The results are shown in Figure 5a,b,c. The numbers shown on the curves refer to the case numbers discussed above.

As might be expected, for case 1 , there is no flow into or out of compartment 2 . In case 2, there will be no flow between compartment 1 and the outside since the door is closed. Figure 5a shows the effect of providing alternate routes for hot gas to leave a space, namely there will be less flow in a given direction as the alternate routes are opened up. The complement to this observation is shown in Figure 5b, namely as flow out of compartment 1 to the outside decreases, and the total flow increases, makeup mass comes from the outside.

The most important and dramatic effect is shown in Figure 5c, which compares the flow out of the upper compartment (2) to the outside. The flow shown here is from the lower layer of the upper compartment to the outside through the window. The lower layer was chosen to show the dramatic and unintuitive flow which results in these four cases. Although gases can escape through ducts, adding a fan to such a configuration has a noticeable effect on the flow and thus could be important in making decisions on whether to use mechanical ventilation to exhaust smoke to aid intervention strategies.

The emphasis in this paper has been on the terms which affect the flow through buildings. These are primarily the radiative and convective heat balance. We have presented the form that the fire takes since it is usually the primary driving term in both the radiation and the convective flow. However, since much of the interest is in the movement of the toxic gases ${ }^{24}$, it is important that the means by which these are generated be made explicit, and we have done so in the section on fire.

\section{Conclusions}

We have presented a refinement of the CFAST model. The work presented in this paper is a significant improvement in these capabilities which allows for a much larger class of structures. As has been shown by Nelson et al. ${ }^{7}$ and Peacock et al. ${ }^{6}$, the predictions of sensible quantities from this model compares favorably with experimental measures of these quantities. As with any theoretical model there are pieces which have been omitted and others which could be implemented more completely. Given the limitations, the model seems to do a credible job. The next steps will be to include a self consistent flame spread model and to reformulate the equations for long corridors where zone models run into difficulty. This latter will involve a term for horizontal momentum. To date we have assumed this is not important. In long corridors the details of the flow are important if we are to model the real world of buildings. 
At the present time, no attempt has been made to ascertain the sensitivity of the specification to results which are obtained. The assumption is that we know the situation we wish to model. Actual use of the model has shown that this assumption is not entirely valid. As a result, we intend to make two improvements in the future. The first is to arrive at an estimate of the sensitivity of output to the specification. This will include geometric effects such as a distribution of door openings and chemical effects such as range for the heat of combustion. Further, we intend to make it possible to run the model automatically for such ranges so that researchers and investigators can ascertain first hand what their assumptions mean.

\section{Acknowledgement}

The work described herein was sponsored in part by the Office of Naval Technology for the Surface Ship Technical Block Program (ONT 211).

\section{Bibliography}

1. Kawagoe, K., Fire Behavior in Rooms, Building Research Institute, Ministry of Construction (Japan), Report No. 27, Tokyo (1958).

2. Tanaka, T., A Mathematical Model of A Compartment Fire, Building Research Institute (Japan) Report 70 (1977), 79 (1978) and 84 (1980).

3. Jones, W. W., A Multicompartment Model for the Spread of Fire, Smoke and Toxic Gases, Fire Safety Journal. 9, 55 (1985).

4. Jones, W. W. and Peacock, R. D., Refinement and Experimental Verification of a Model for Fire Growth and Smoke Transport, Proceedings of the Second International Symposium on Fire Safety Science, Tokyo (1988).

5. Jones, W. W. and Forney, G. P., A Programmer's Reference Manual for CFAST, the Unified Model of Fire Growth and Smoke Transport, National Institute of Standards and Technology Technical Note 1283 (1990).

6. Peacock, R. D., Jones, W. W. and Bukowski, R. W., Verification of a Model of Fire and Smoke Transport, submitted to the Fire Safety Journal (1992).

7. Nelson, H. E. and Deal, S., Comparing Compartment Fires with Compartment Fire Models, Proceedings of the Third International Symposium on Fire Safety Science, 719 (1991).

8. Bukowski, R. W., An Analysis of the Happyland Social Club Fire with HAZARD I, Fire and Arson Investigator 42, 3(1992).

9. Brenan, K. E., Campbell, S. L., and Petzold, L. R., Numerical Solution of Initial Value Problems in Differential Algebraic Equations, North-Holland, New York (1989).

10. Zwillinger, D., Handbook of Differential Equations, Academic Press, New York (1989). 
11. Cooper L. Y., and Forney, G. P., The consolidated compartment fire model (CCFM) computer application CCFM-VENTS - part i: Physical reference guide. National Institute of Standards and Technology, NISTIR 4342 (1990).

12. Forney, G. P., and Moss, W. F., Analyzing and Exploiting Numerical Characteristics of Zone Fire Models, National Institute of Standards and Technology, NISTIR 4763 (March 1992).

13. Jones, W. W. and Bodart, X., Buoyancy Driven Flow as the Forcing Function of Smoke Transport Models, National Institute of Standards and Technology, Internal Report NBSIR 86-3329 (1986).

14. Jones, W. W., Refinement of a Model for Fire Growth and Smoke Transport, NIST Technical Note 1282 (1990).

15. Jones, W. W. and Peacock, R. D., Technical Reference Guide for FAST Version 18, NIST Technical Note 1262 (1989).

16. Quintiere, J. G., Steckler, K., and Corley, D., An Assessment of Fire Induced Flows in Compartments, Fire Science and Technology 4, 1 (1984).

17. Cooper, L. Y., Calculation of the Flow Through a Horizontal Ceiling/Floor Vent, National Institute of Standards and Technology IR 89-4052 (1989).

18. Klote, J. H., A Computer Model of Smoke Movement by Air Conditioning Systems, NBSIR 87-3657 (1987).

19. Forney, G. P., Computing Radiant Heat Transfer Occurring in a Zone Fire Model, to be published (1991).

20. Siegel, R. and Howell, J. R., Thermal Radiation Heat Transfer, Hemisphere Publishing Corporation, New York, second ed. (1981).

21. Hottel, H. C., Heat Transmission, McGraw-Hill Book Company, New York, third ed. (1954).

22. Hottel, H. and Cohen, E., Radiant Heat Exchange in a Gas Filled Enclosure: Allowance for non-uniformity of Gas Temperature, American Institute of Chemical Engineering Journal 4, 3 (1958).

23. Yamada, T. and Cooper, L. Y., Algorithms for Calculating Radiative Heat Exchange Between the Surfaces of an Enclosure, the Smoke Layers and a Fire, Building and Fire Research Laboratory Research Colloquium, July 20, 1990.

24. Babrauskas, V., Levin, B. C., Gann, R. G., Paabo, M., Harris, Jr., R. H., Peacock, R. D., Yusa, S., Toxic Potency Measurement for Fire Hazard Analysis, NIST Special Publication, in press, 1991. 


\section{Figure Captions}

Figure 1. Exchange flow through a ceiling vent.

Figure 2. Net radiation at the $k^{\prime}$ th segment.

Figure 3. Comparison of radiation flux from the ceiling and upper wall.

Figure 4. Geometry of the compartments for case 1 through 4.

Figure 5a Flow from compartment 1 to the outside (case 1, 3 and 4).

Figure 5b Flow from the outside into the lower layer of compartment 1.

Figure 5c Flow from compartment 2 to the outside.

\section{Nomenclature}

m mass in kilograms

$\dot{m}$ rate of mass change in kilograms per second

$\dot{\mathrm{E}} \quad$ defined quantity - total enthalpy $(\dot{\mathrm{Q}}+\dot{\mathrm{h}})$

e internal energy in joules per cubic meter

$\mathrm{V}$ volume in cubic meters

$P \quad$ pressure in newtons per square meter

R gas constant (239 joules per kilogram per kelvins for air)

$\mathrm{T}$ temperature in kelvins

c specific heat (subscript $v$ for constant volume and subscript $p$ for constant pressure)

c heat source in the radiation equation, indexed by gas layer and fires

$\mathrm{h}$ energy of formation (used only in eq? when subscripted with ' $i, o^{\prime}$ heat of combustion when subscripted with ' $c$ '

$\mathrm{h}$ enthalpy flux in watts

Q rate of change of energy (watts)

$t$ time in seconds

$\dot{s}$ defined quantity - sum of $\dot{E}$ 's

$S \quad$ width of an opening (vent) in meters

$\gamma \quad$ ratio of specific heat $c_{\mathrm{p}} / \mathrm{c}_{\mathrm{v}}$

$\beta \quad \gamma /(\gamma-1)$

A area in square meters

C flow coefficient, typically 0.65 to 0.75 for the types of openings used

q heat flux in watts per square meter

$\Delta \quad$ change in a quantity

$\varepsilon \quad$ emissivity, expansion variable for pressure - both dimensionless; see text for usage

$\sigma \quad$ Stefan-Boltzman constant $\left(5.67 \times 10^{-8}\right.$ watts/square meter $/$ kelvins $\left.{ }^{4}\right)$

$\rho \quad$ mass density in kilograms per cubic meter

F configuration (view) factor for radiation friction factor for flow through ducts

f variable flow coefficient for vertical flow (function, not indexed) sum of mass flow around a closed node loop (indexed)

D equivalent diameter for a duct - used in matching real duct openings

G conductance of a duct, the inverse of the resistance 
g gravitational constant, 9.8 meters per second

v velocity in meters per second

$z \quad$ height in meters

$\mathrm{x}, \mathrm{y}$ intermediate variables

Subscripts:

R reference

c convective

$\mathrm{i}, \mathrm{j}$ compartment indices

f fire

p pressure ( $c_{p}$ for specific heat at constant pressure) and pyrolysis

$\mathrm{u}, 1$ upper or lower layer, respectively ( $\mathrm{k}$ is used as an index over $\{\mathrm{u}, 1\}$ )

$v \quad$ volume ( $c_{v}$ which is the specific heat at constant volume) and volatilization

a ambient

k surface index

$1,2, .$. height numbering scheme

$\mathrm{i}, \mathrm{o}$ used together as compartment on the inside to compartment on the outside

av average

e equivalent

0 initial 


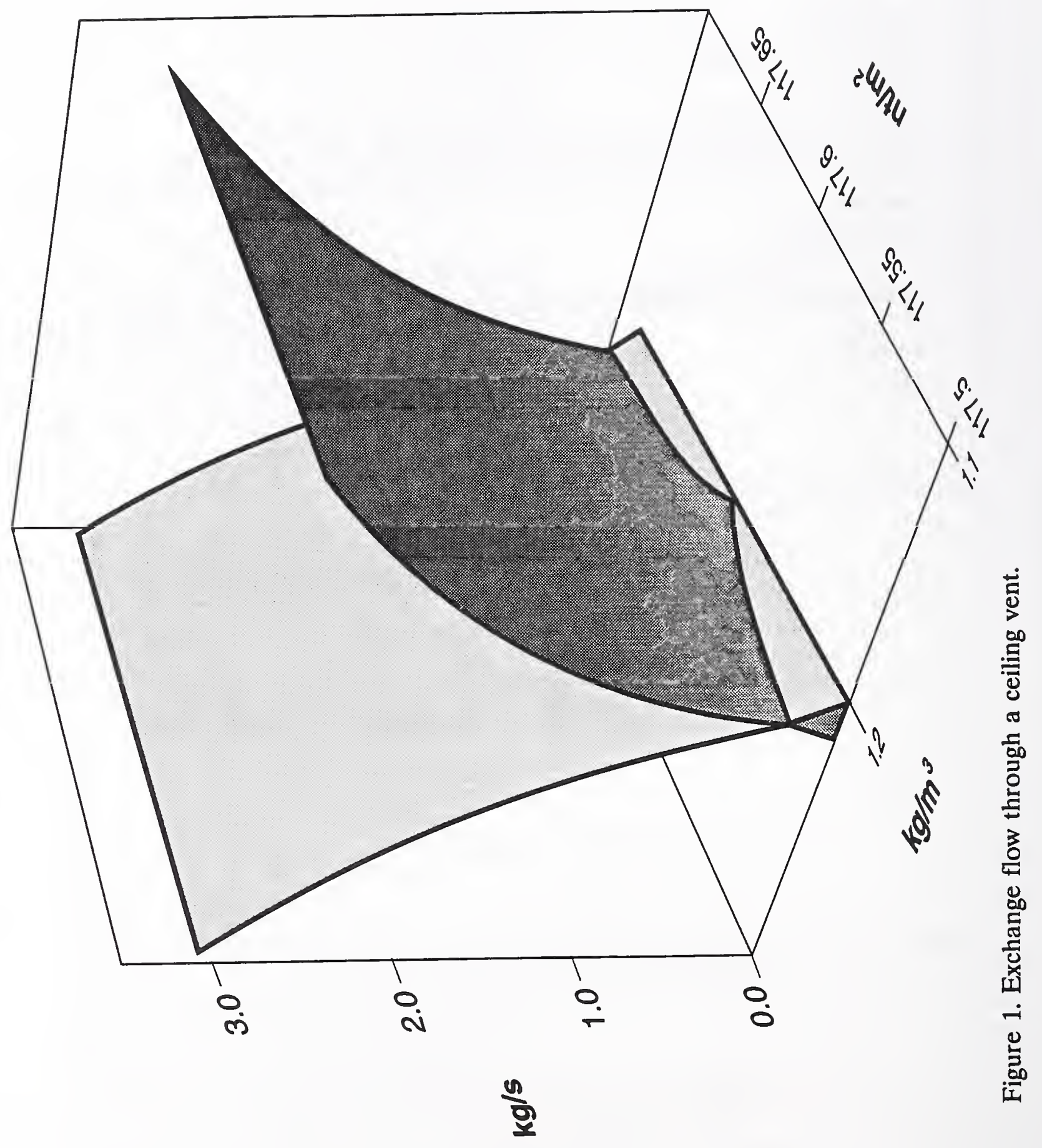




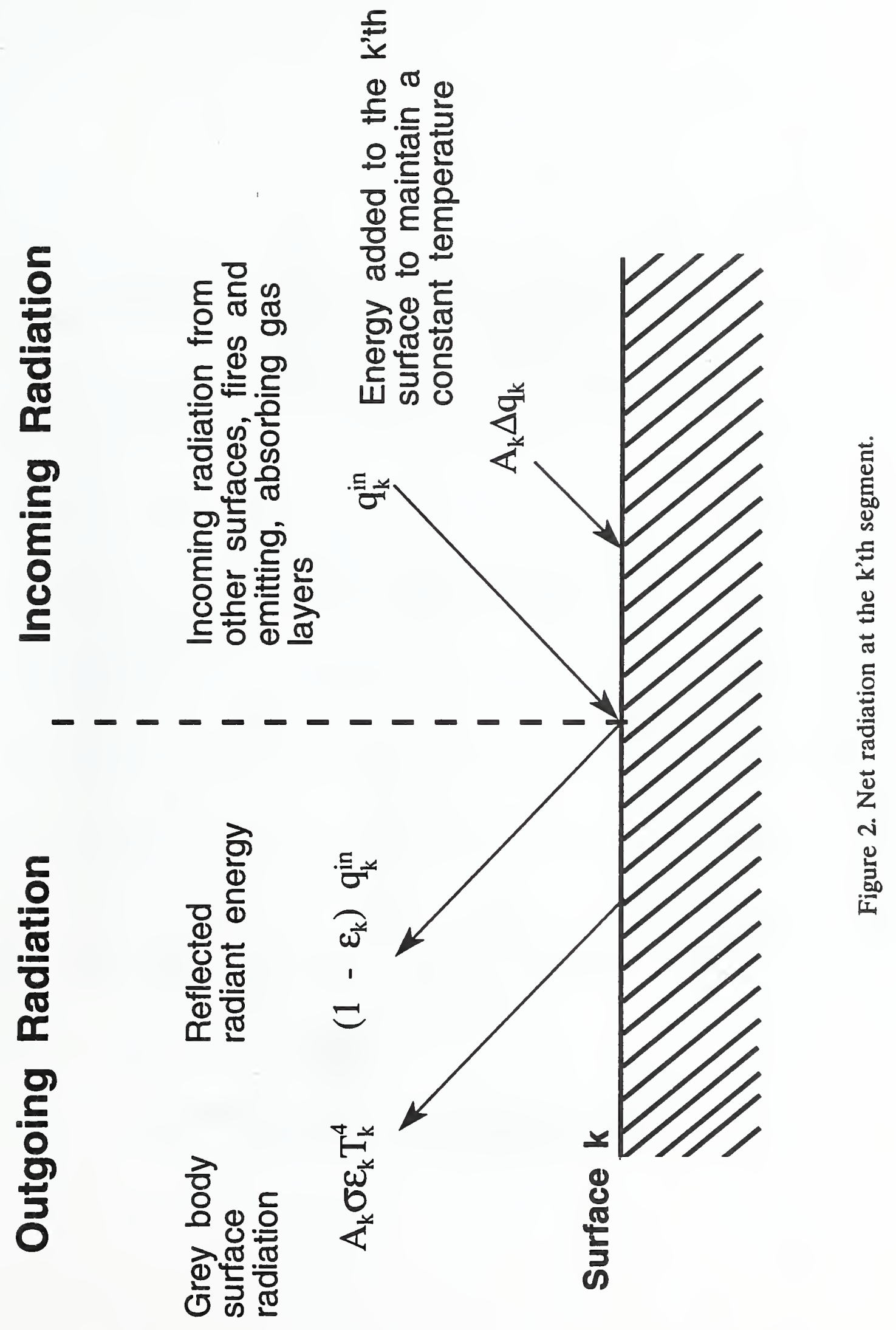




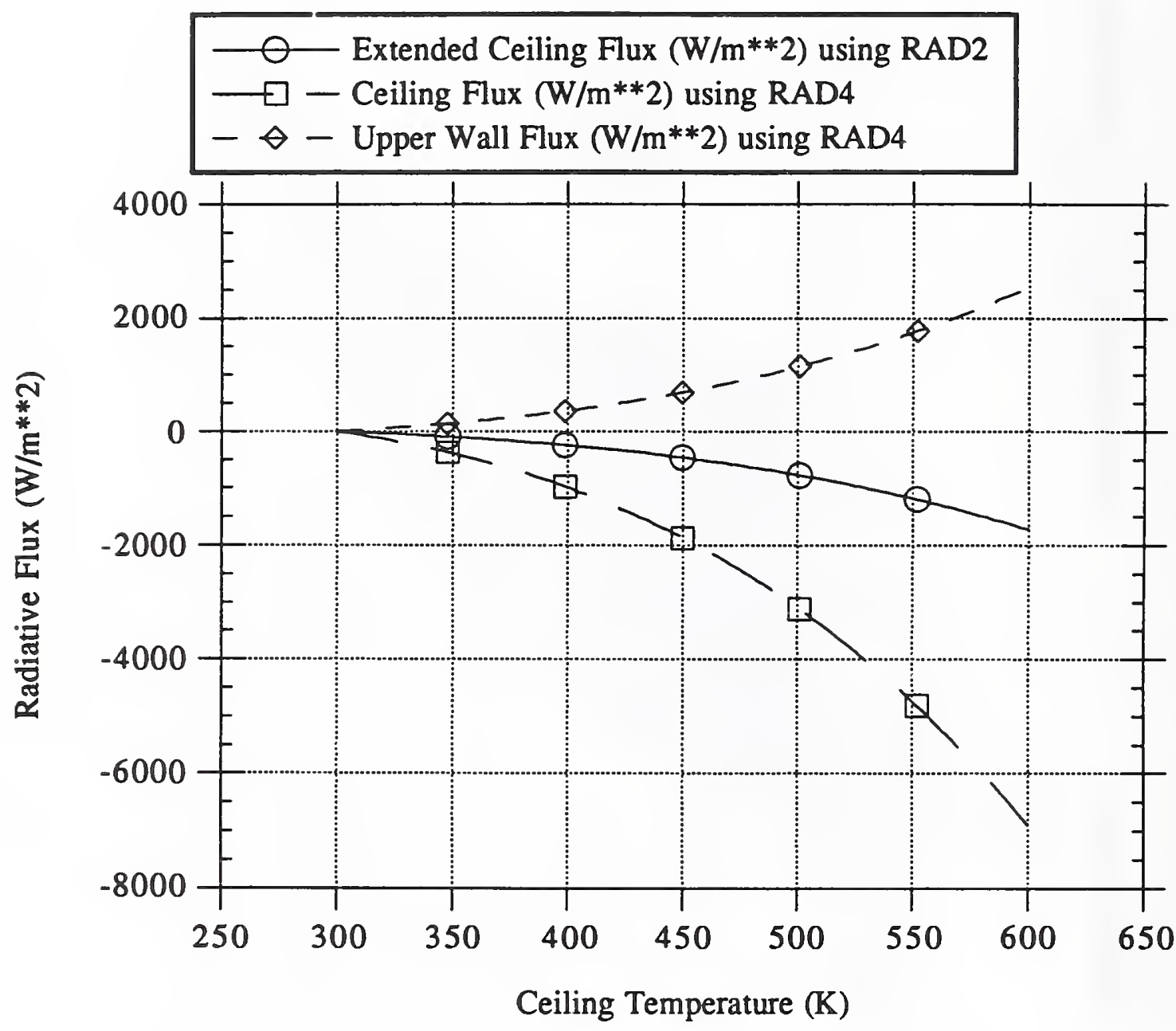

Figure 3. Comparison of radiation flux from the ceiling and upper wall. 


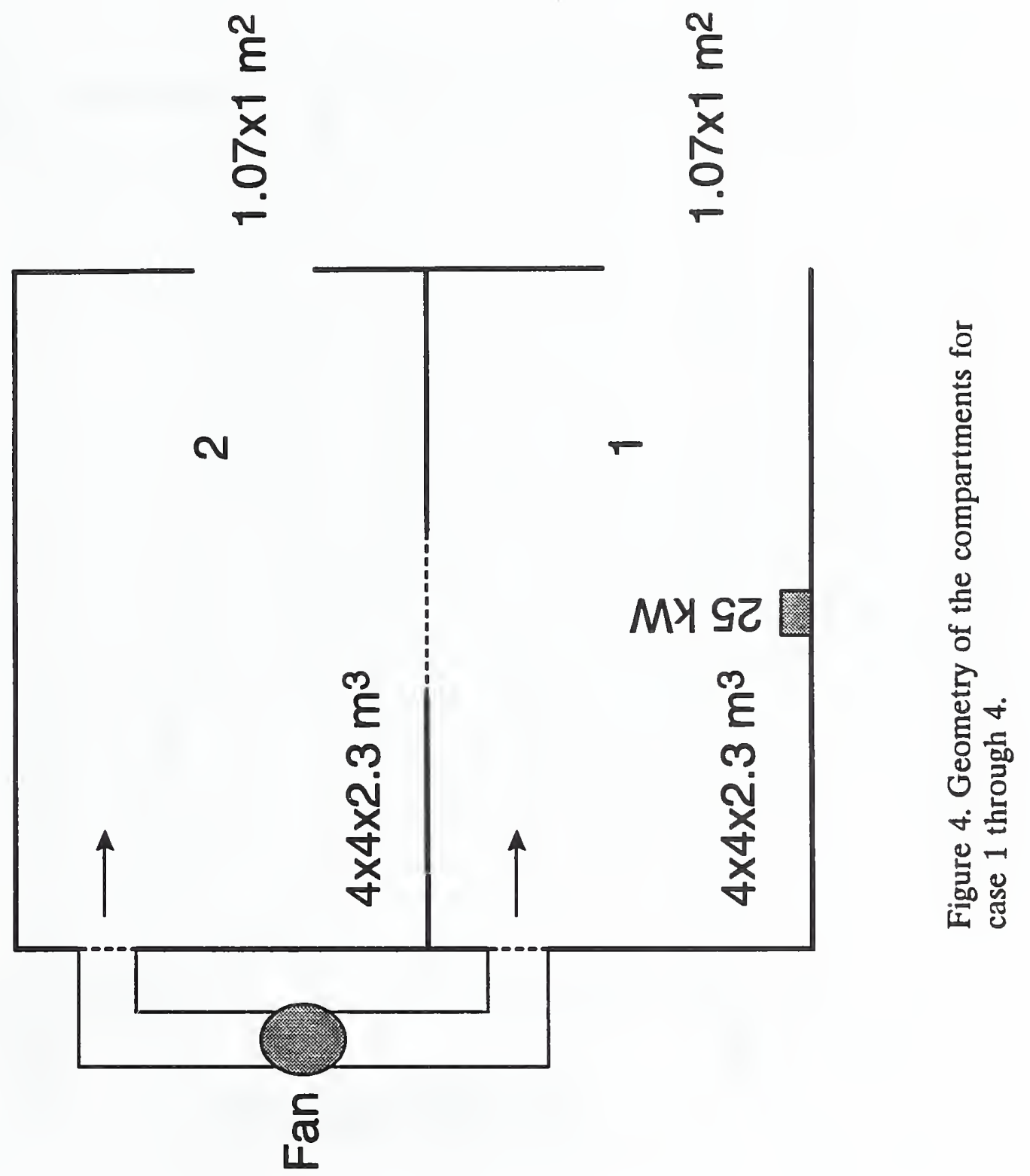




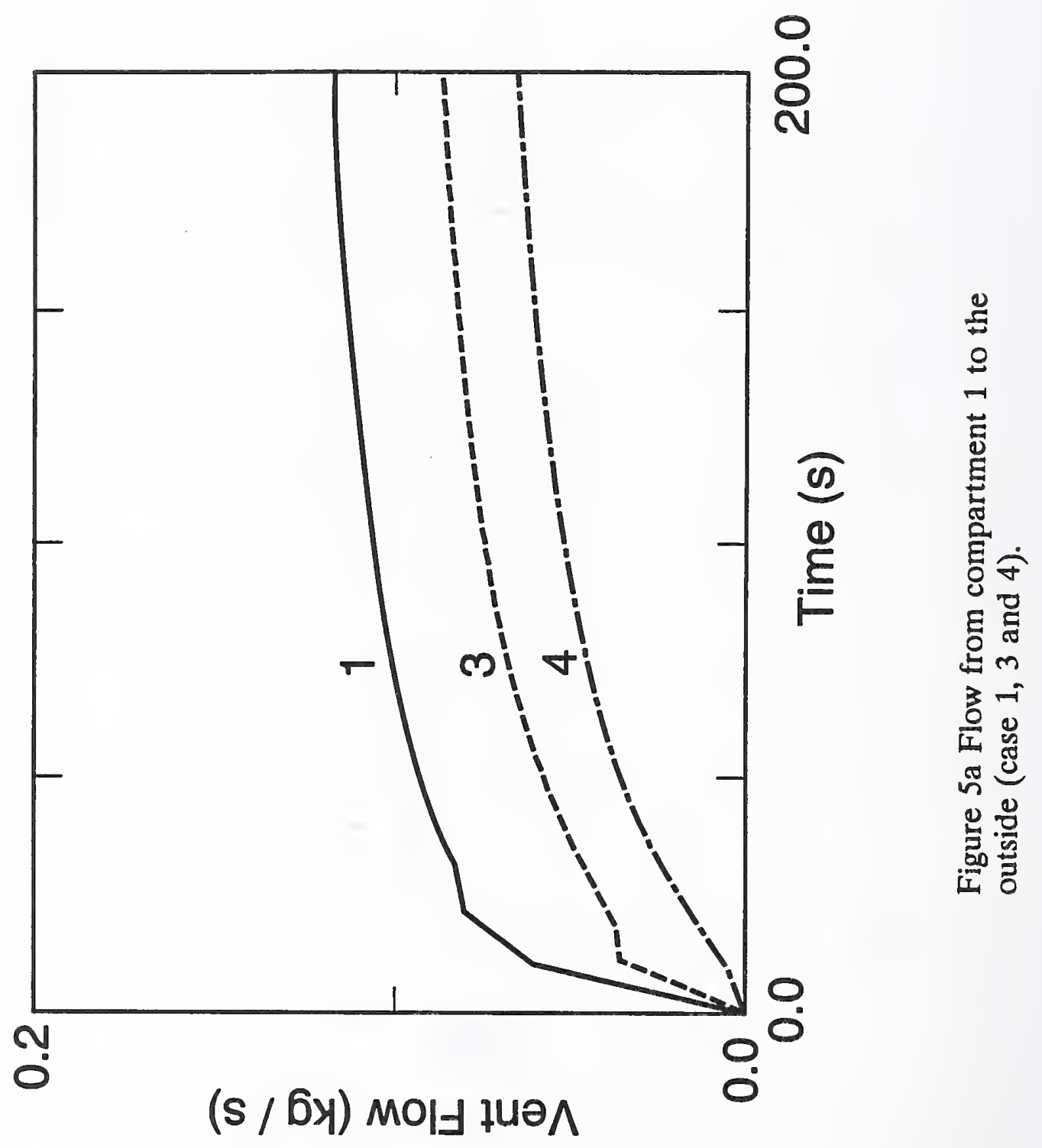




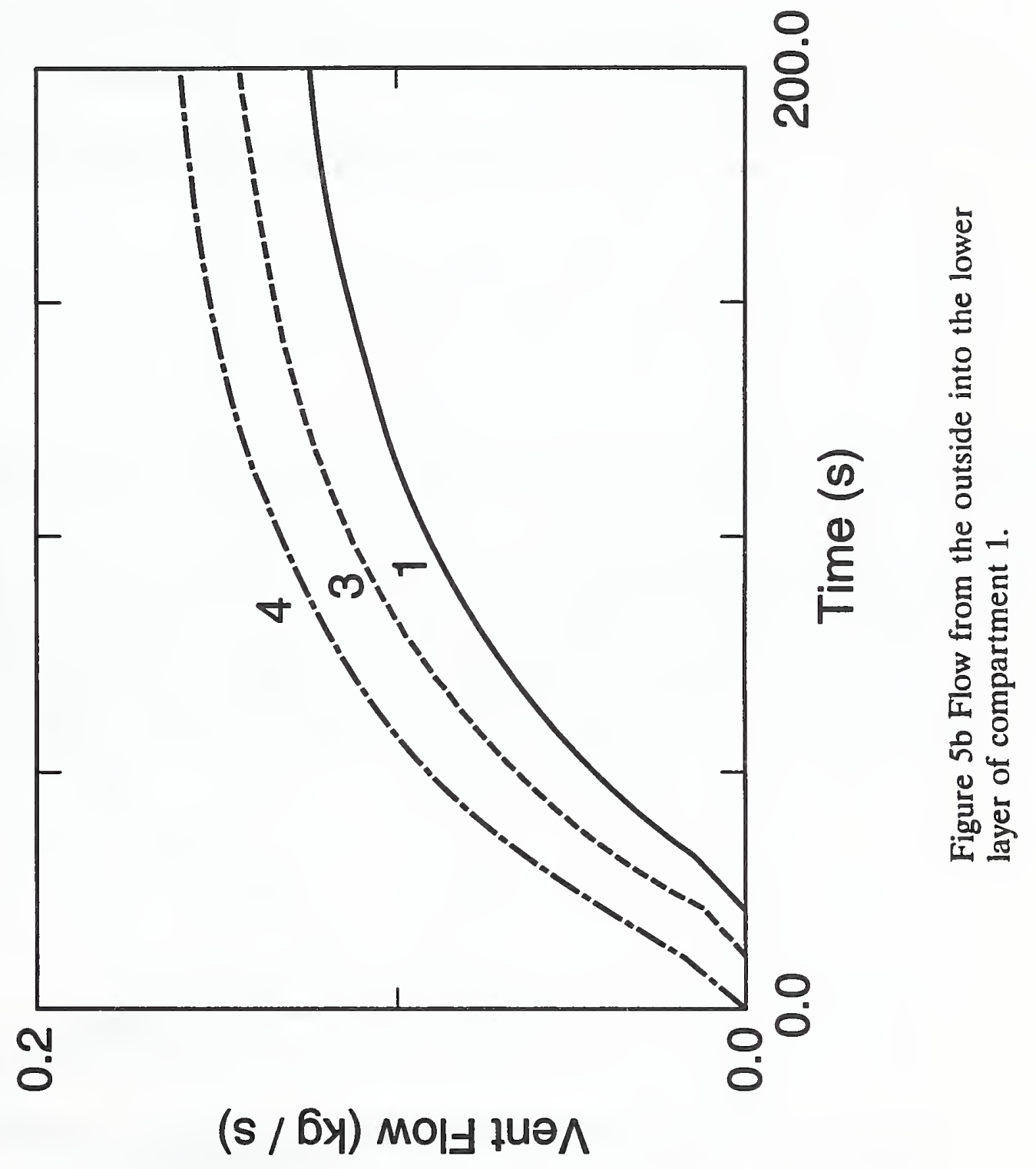




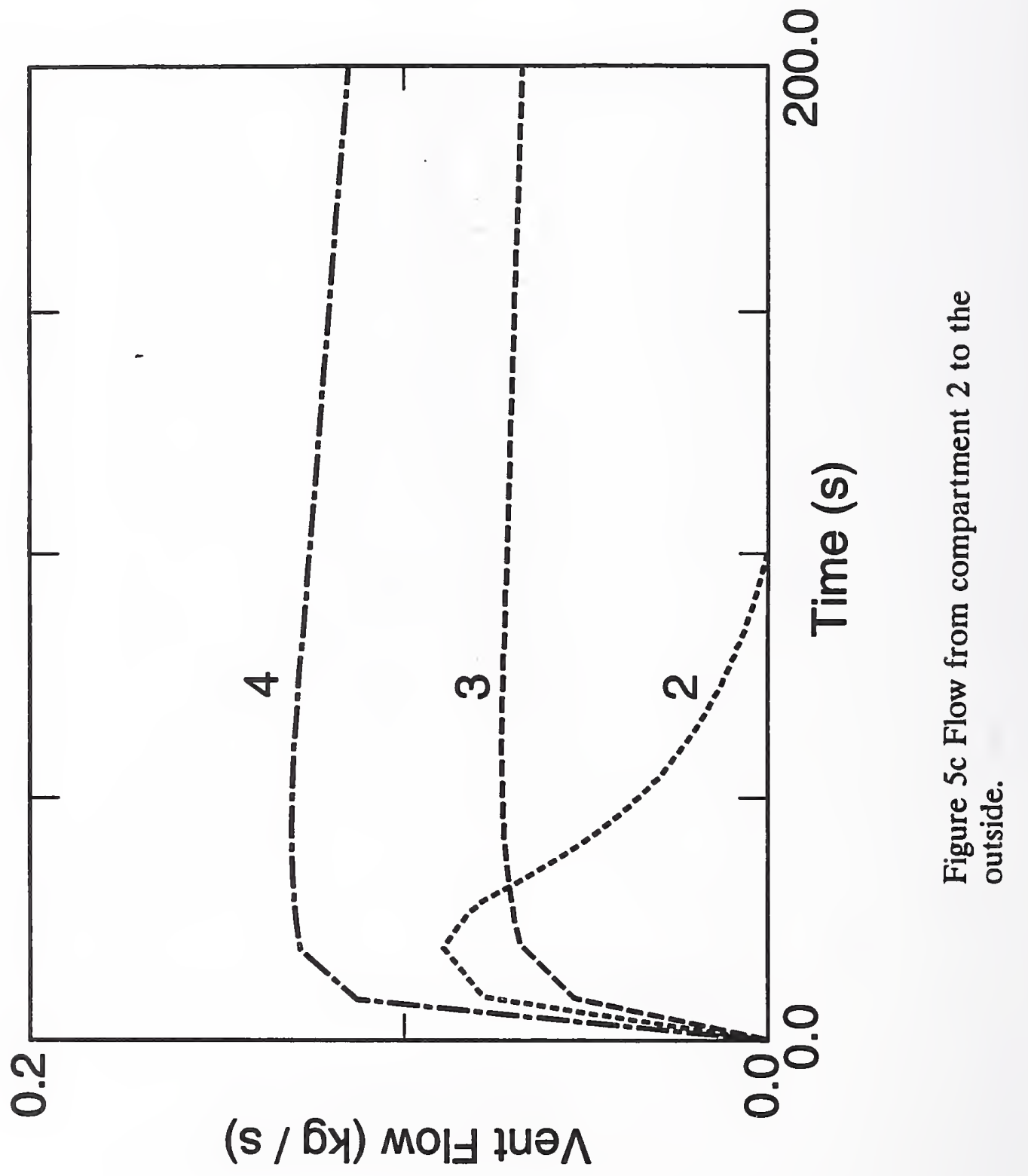



